\title{
Spherical-Wave Based Triangular Finite Element Models for Axial Symmetric Helmholtz Problems
}

\author{
G.H.Liu ${ }^{1}$, Q.H.Zhang ${ }^{2,1}$, K.Y.Sze ${ }^{1}$ \\ 1: Department of Mechanical Engineering, The University of Hong Kong, Pokfulam, Hong Kong SAR, P.R.China. \\ 2: Department of Scientific Computing \& Computer Applications, Sun Yat-Sen University, Guangzhou, P.R. China. \\ *Correspondence author (email: kysze@ hku.hk)
}

\begin{abstract}
In this paper, six-node hybrid triangular finite element models are devised for axial symmetric Helmholtz problems. In the formulation, boundary and domain approximations to the Helmholtz field are defined for each element. While the boundary approximation is constructed by nodal interpolation, the domain approximation satisfies the Helmholtz equation and is composed of spherical waves with source points located along the axis of symmetry. To formulate rank sufficient six-node elements, a minimal of six wave modes from three source points are required. Two methods of selecting the source points are attempted. In the first method, the directions of the waves passing through the element are essentially parallel to the three lines connecting the parametric centre of the element and its three corner (or side) nodes. In the second method, the directions are essentially equally spaced at $2 \pi / 3$ interval in the r-z-plane. For the attempted examples, the average error ratios of the proposed elements and the conventional element are around $50 \%$.
\end{abstract}

Keywords: triangular, axisymmetric, Helmholtz, hybrid, spherical waves, finite element, Trefftz

Published as Finite Elements in Analysis \& Design 47: 342-350 (2011)

doi:10.1016/j.finel.2010.12.002 


\section{INTROUDCTION}

In the hybrid finite element method for stress/structural analyses, the displacement-based finite element models are enhanced by introducing stress, strain or another displacement as the additional field variable(s) to the displacement approximation constructed by nodal interpolation [1-10]. In the case of the hybrid-displacement method, the additional field is a domain displacement which leads to equilibrating stress and may also satisfy some homogeneous boundary conditions $[2,3,9,10]$. This category of hybrid elements are also known as hybrid-Trefftz or Trefftz elements linked by the displacement-frame or the boundary displacement [4,6-10]. The underlying reason is that the domain displacement is mainly truncated from a Trefftz solution set which is the basis of the Trefftz nonsingular boundary element methods.

A major challenge in finite element analyses of Helmholtz problems is that the solutions are spatially oscillating throughout the entire problem domains. While considerable computational saving can be realized by using graded meshes in stress analyses, the practice is not applicable to Helmholtz problems. Hence, the mesh requirement induces tremendous computing load when the wavenumber or the problem domain size increases. To better tackle the issue, a number of wave-based approaches that make use of solution sets for the wave or Helmholtz equations have been proposed in the last decades. These include the Trefftz methods [11-18], the plane-wave basis method [19-22] and the discontinuous enrichment method [23,24], among others.

Though a number of Trefftz boundary element methods have been formulated for Helmholtz problems [11-15], Trefftz finite element models do not appear to be abundant. Among them, the least-square models [16,17] and the traction-frame models [18] can be noted. All Trefftz models possess their own domain approximations which are extracted from Trefftz solution sets. In the plane-wave basis method, the plane wave solutions are employed as the nodal enrichment functions in the context of the partition of unity finite element method [19-22]. The value of the Helmholtz variable at a node is the sum of plane wave solutions which represent plane waves propagating along different directions. Within the element, the Helmholtz variable is obtained by the conventional nodal interpolation. Thus, the system equation unknowns are the amplitudes of the plane waves at the nodes but not the nodal value of the Helmholtz variable. In the discontinuous enrichment method, the coarse scale approximation constructed by the conventional nodal interpolation is enriched by plane wave solutions. The enrichment which is intended to resolve the fine scale phenomenon induces discontinuity across the inter-element boundary [23,24]. Weak enforcement of the continuity is implemented through Lagrange multipliers. While the fine scale enrichments can be condensed at element level, the multipliers which link the enrichments of adjacent elements enter the global 
equation.

In this paper, hybrid-Trefftz six-node triangular elements will be formulated for the axial symmetric Helmholtz problem for which there are hardly any advanced finite element models. Unlike the previous Trefftz finite elements, the present ones can readily be incorporated into the standard finite element program framework. Independent boundary and domain approximations to the Helmholtz field are defined. The boundary approximation is constructed by nodal interpolation. Equality of the two approximations is enforced along the element boundary [25-27]. Indeed, the hybrid variational functional employed in the formulation is similar to the functional used in elasticity hybrid-Trefftz elements with displacement-frame $[2-4,8-10,18]$. The spherical wave solutions are employed to construct the domain approximation. For rank sufficiency, a six-node element has to be equipped with at least six wave modes from three source points. Two methods of selecting the source points are attempted. In the first method, the directions of the waves passing through the element are essentially parallel to the three lines connecting the parametric centre of the element and its three corner (or side) nodes. In the second method, the directions are essentially equally spaced at $2 \pi / 3$ interval. For the attempted examples, the average error ratios of the proposed elements and the conventional element are around $50 \%$ at considerably dense meshes.

\section{CONVENTIONAL FORMULATION}

Helmholtz equation is often introduced by using the steady state acoustics. The Helmholtz variable $u$ can be the spatial amplitude of the acoustic pressure or the velocity potential. This paper will restrict itself to bounded domains. Under the axial symmetry, a problem domain $\Omega$ is often considered thru its cross-section area $A$ in the $r$-z-plane where $r \geq 0$, see Figure 1 . When $A$ is discretized into subareas or finite elements $A^{e}$ s, the problem can be summarized as:

(a) Helmholtz equation: $\nabla^{2} u+k^{2} u=0 \quad$ in all $A^{e} \mathrm{~s}$

(b) natural boundary condition: $\widehat{\mathbf{n}}^{T} \hat{\nabla} u=\bar{t} \quad$ on all $\Gamma_{n}^{e}$

(c) essential boundary condition: $u=\bar{u}$ and $\delta u=0 \quad$ on all $\Gamma_{u}^{e}$

(d) natural interfacial condition: $\left(\hat{\mathbf{n}}^{T} \widehat{\nabla} u\right)^{+}+\left(\hat{\mathbf{n}}^{T} \widehat{\nabla} u\right)^{-}=0 \quad$ on all $\Gamma_{m}^{e}$

(e) essential interfacial condition: $u^{+}=u^{-}$and $\delta u^{+}=\delta u^{-} \quad$ on all $\Gamma_{m}^{e}$

In the above expressions, $\nabla^{2}$ is the Laplace operator (see Appendix), $\hat{\nabla}=(\partial / \partial r, \partial / \partial z)^{T}$, $\widehat{\mathbf{n}}=\left(\cos \theta_{n}, \sin \theta_{n}\right)^{T}$ where $\theta_{n}$ is the inclination of the outward normal vector of the element boundary 
to the $r$-axis, $k$ is the wavenumber, $\delta$ is the variational symbol and $\Gamma_{m}^{e}$ is the inter-element boundary. Moreover, ()$^{+}$and ()$^{-}$denote the braced quantities at the two sides of $\Gamma_{m}^{e}$. In the absence of dissipation, $k$ is real. Otherwise, it is complex. For simplicity, it will be assumed as usual that element boundary $\partial A^{e}$ can be partitioned into the non-overlapping portions $\Gamma_{t}^{e}, \Gamma_{u}^{e}$ and $\Gamma_{m}^{e}$, i.e.

$$
\Gamma_{t}^{e} \cup \Gamma_{u}^{e} \cup \Gamma_{m}^{e}=\partial A^{e} \text { and } \Gamma_{t}^{e} \cap \Gamma_{u}^{e}=\Gamma_{u}^{e} \cap \Gamma_{m}^{e}=\Gamma_{m}^{e} \cap \Gamma_{t}^{e}=\text { null. }
$$

The terms "natural interfacial condition" and "essential interfacial condition" are not widely used. However, they are indeed the interfacial counterparts of the natural and essential boundary conditions, respectively.

The elemental variational functional for the conventional finite element formulation of the Helmholtz problem is known to be:

$$
\Pi^{e}=\frac{1}{2} \int_{\Omega^{e}}\left[(\nabla u)^{T} \nabla u-k^{2} u^{2}\right] d \Omega-\int_{S_{t}^{e}} \bar{u}_{n} u d S
$$

where $u$ satisfies the essential boundary and continuity conditions. Under the axial symmetry, the differential volume $d \Omega$ and differential surface area $d S$ can be replaced by respectively $2 \pi r d A$ and $2 \pi r d \Gamma$ in which the common factor $2 \pi$ can be neglected for simplicity. Then, the functional becomes

$$
\Pi^{e}=\frac{1}{2} \int_{A^{e}}\left[(\nabla u)^{T} \nabla u-k^{2} u^{2}\right] r d A-\int_{\Gamma_{t}^{e}} \bar{u}_{n} u r d \Gamma .
$$

For any smooth axial symmetric functions $f=f(r, z)$ and $h=h(r, z)$, the divergence theorem can be read (see Appendix) as:

$$
\int_{A^{e}}\left[(\nabla h)^{T}(\nabla f)+h \nabla^{2} f\right] r d A=\oint_{\partial A^{e}} h\left(\hat{\mathbf{n}}^{T} \hat{\nabla} f\right) r d \Gamma
$$

With $h$ and $f$ taken to be respectively $\delta u$ and $u$ and recalling that $\delta u=0$ on $\Gamma_{u}^{e}$, variation of (3) is:

$$
\delta \Pi^{e}=-\int_{A^{e}} \delta u\left(\nabla^{2} u+k^{2} u\right) r d A+\int_{\Gamma_{t}^{e}} \delta u\left(\widehat{\mathbf{n}}^{T} \hat{\nabla} u-\bar{u}_{n}\right) r d S+\int_{\Gamma_{m}^{e}} \delta u\left(\hat{\mathbf{n}}^{T} \hat{\nabla} u\right) r d S .
$$

It can be seen that the first integral enforces (a), the second integral enforces (b) and the third integral, when $\delta \Pi^{e}$ s of the adjacent elements are assembled, enforces (d).

Figure 2(a) shows the six-node triangular element in the global $r$ - $z$-plane. To formulate a conventional element, $r, z$ and $u$ are obtained by interpolation which can be expressed as: 


$$
r=\sum_{i=1}^{6} N_{i} r_{i}, z=\sum_{i=1}^{6} N_{i} z_{i} \text { and } u=\sum_{i=1}^{6} N_{i} u_{i}=\left[N_{1}, \ldots, N_{6}\right]\left\{\begin{array}{c}
u_{1} \\
\vdots \\
u_{6}
\end{array}\right\}=\mathbf{N d}
$$

where $N_{1}=(1-s-t)(1-2 s-2 t), N_{2}=t(2 t-1), N_{3}=s(2 s-1), N_{4}=4 s t, N_{5}=4 s(1-s-t), N_{6}=4 t(1-s-t)$ and $(s, t)$ are the area coordinates which vary between 0 and 1 . The interpolation matrix $\mathbf{N}$ and the vector of nodal dofs $\mathbf{d}$ are self-defined. The $\mathrm{C}^{0}$ continuity nature of $\mathbf{N}$ can readily enable the satisfaction of (c) and (d). With (6) substituted into (3), the latter becomes

$$
\Pi^{e}=\frac{1}{2} \mathbf{d}^{T} \mathbf{K}_{c} \mathbf{d}-\mathbf{f}^{T} \mathbf{d}
$$

where

$$
\mathbf{K}_{c}=\int_{A^{e}}\left[(\nabla \mathbf{N})^{T}(\nabla \mathbf{N})-k^{2} \mathbf{N}^{T} \mathbf{N}\right] r d A=\int_{0}^{1} \int_{0}^{1-t}\left[(\nabla \mathbf{N})^{T}(\nabla \mathbf{N})-k^{2} \mathbf{N}^{T} \mathbf{N}\right] r J d s d t \text { and } \mathbf{f}=\int_{\Gamma_{t}^{e}} \mathbf{N}^{T} \bar{u}_{n} r d \Gamma .
$$

Furthermore, $J=(\partial r / \partial s)(\partial \mathrm{z} / \partial t)-(\partial z / \partial s)(\partial r / \partial t)$ is the Jacobian determinant of the coordinate transformation between $(r, \mathrm{z})$ and $(s, t)$.

As $\mathbf{N}$ is quadratic in $(s, t)$, the six-point integration rule which is exact up to the fourth order is required to evaluate the element.

\section{HYBRID-TREFFTZ FINITE ELEMNET FORMULATION}

The following elemental hybrid functional can be formed by introducing a second Helmholtz approximation $g$ to (3) [25-27]:

$$
\Pi_{h}^{e}=\frac{1}{2} \int_{A^{e}}\left[(\nabla g)^{T} \nabla g-k^{2} g^{2}\right] r d A+\int_{\partial A^{e}}\left(\hat{\mathbf{n}}^{T} \hat{\nabla} g\right)(u-g) r d \Gamma-\int_{\Gamma_{t}^{e}} \bar{t} u r d \Gamma
$$

from which

$$
\delta \Pi_{h}^{e}=\int_{A^{e}}\left[(\nabla g)^{T} \nabla \delta g-k^{2} g \delta g\right] r d A+\int_{\partial A^{e}}\left[\left(\hat{\mathbf{n}}^{T} \hat{\nabla} \delta g\right)(u-g)+\left(\hat{\mathbf{n}}^{T} \hat{\nabla} g\right)(\delta u-\delta g)\right] r d \Gamma-\int_{\Gamma_{t}^{e}} \bar{t} \delta u \cdot r d \Gamma .
$$

With $h$ and $f$ in (4) taken to be respectively $g$ and $\delta g$, (9) can be expressed as:

$$
\begin{aligned}
\delta \Pi_{h}^{e}= & -\int_{A^{e}}\left(\nabla^{2} g+k^{2} g\right) \delta g \cdot r d A+\int_{\partial A^{e}}\left(\hat{\mathbf{n}}^{T} \hat{\nabla} \delta g\right)(u-g) r d \Gamma \\
& +\int_{\Gamma_{t}^{e}}\left(\hat{\mathbf{n}}^{T} \hat{\nabla} g-\bar{t}\right) \delta u \cdot r d \Gamma+\int_{\Gamma_{m}^{e}}\left(\hat{\mathbf{n}}^{T} \hat{\nabla} g\right) \delta u \cdot r d \Gamma .
\end{aligned}
$$

The first to the fourth integral enforce (a), the equality of $u$ and $g$, (b) and (d), respectively. Same as the conventional formulation, when $u$ is obtained by nodal interpolation, (c) and (e) can be ensured 
readily. If $g$ satisfies the Helmholtz equation strictly, i.e. $\nabla^{2} g+k^{2} g=0$, the area integration in functional can be avoided by virtue of (4) and

$$
\Pi_{h}^{e}=\int_{\partial A^{e}}\left[-\frac{1}{2}\left(\hat{\mathbf{n}}^{T} \hat{\nabla} g\right) g+\left(\hat{\mathbf{n}}^{T} \widehat{\nabla} g\right) u\right] r d \Gamma-\int_{\Gamma_{t}^{e}} \bar{t} u \cdot r d \Gamma .
$$

Now, $u$ only has to be known along element boundary and it can therefore be termed as the boundary approximation. On the other hand, $g$ satisfies the domain condition and must be known within the element domain. It can be therefore termed as the domain approximation and expressed as:

$$
g=\mathbf{P} \boldsymbol{\beta}
$$

where $\mathbf{P}$ is the shape function matrix and $\boldsymbol{\beta}$ is the vector of coefficients. With (6) and (12) substituted into (11),

$$
\Pi_{h}^{e}=-\frac{1}{2} \boldsymbol{\beta}^{T} \mathbf{H} \boldsymbol{\beta}+\boldsymbol{\beta}^{T} \mathbf{G} \mathbf{d}-\mathbf{f}^{T} \mathbf{d}
$$

in which

$$
\mathbf{H}=\int_{\partial A^{e}}\left(\hat{\mathbf{n}}^{T} \widehat{\nabla} \mathbf{P}\right)^{T} \mathbf{P} r d \Gamma \text { and } \mathbf{G}=\int_{\partial A^{e}}\left(\hat{\mathbf{n}}^{T} \hat{\nabla} \mathbf{P}\right)^{T} \mathbf{N} \cdot r d \Gamma .
$$

$\mathbf{H}$ should be symmetric but its evaluation by numerical integration induces asymmetry. By recalling the divergence theorem in Appendix and $\nabla^{2} \mathbf{P}+k^{2} \mathbf{P}=0, \mathbf{H}$ and $\mathbf{G}$ can be expressed as:

$$
\mathbf{H}=\int_{A^{e}}\left[(\nabla \mathbf{P})^{T}(\nabla \mathbf{P})-k^{2} \mathbf{P}^{T} \mathbf{P}\right] r d A \text { and } \mathbf{G}=\int_{A^{e}}\left[(\nabla \mathbf{P})^{T}(\nabla \mathbf{N})-k^{2} \mathbf{P}^{T} \mathbf{N}\right] r d A .
$$

Domain integration will be employed to avoid the asymmetry of $\mathbf{H}$.

As there is no inter-element continuity requirement on $g, \boldsymbol{\beta}$ can be condensed from (13) thru the stationary condition $\mathbf{H} \boldsymbol{\beta}=\mathbf{G d}$ of the functional. The latter becomes:

$$
\Pi_{h}^{e}=\frac{1}{2} \mathbf{d}^{T}\left(\mathbf{G}^{T} \mathbf{H}^{-1} \mathbf{G}\right) \mathbf{d}-\mathbf{f}^{T} \mathbf{d}=\frac{1}{2} \mathbf{d}^{T} \mathbf{K}_{h} \mathbf{d}-\mathbf{f}^{T} \mathbf{d}
$$

where $\mathbf{K}_{h}$ is self-defined and is the element matrix of the hybrid-Trefftz finite element model. Same as $\mathbf{K}_{c}, \mathbf{K}_{h}$ can also be incorporated into the standard finite element program framework. Boundary conditions for $\mathbf{K}_{h}$ and $\mathbf{K}_{c}$ are prescribed in identical manner.

In order that the element matrix $\mathbf{K}_{h}$ is rank sufficient or has full rank, it can be proven by simple matrix algebra that $\operatorname{dim} .(\boldsymbol{\beta}) \geq \operatorname{dim} .(\mathbf{d})$. 


\section{SPHERICAL WAVES AS DOMAIN MODES}

While the above section outlines the principle to formulate the hybrid-Trefftz element for the axial symmetric Helmholtz problem, elements with their domain approximation modes devised from the spherical wave solution will be presented in this section. Let $S\left(0, z_{S}\right)$ be the source point (see Figure 1), the spherical waves are

$$
\left\{\frac{\exp \left(i k R_{S}\right)}{k R_{S}}, \frac{\exp \left(-i k R_{S}\right)}{k R_{S}}\right\} \text { or }\left\{\frac{\cos \left(k R_{S}\right)}{k R_{S}}, \frac{\sin \left(k R_{S}\right)}{k R_{S}}\right\}
$$

where $R_{S}^{2}=r^{2}+\left(z-z_{S}\right)^{2}$ is the square of distance between the source point $\mathrm{S}$ and $(r, z)$.

To formulate rank sufficient six-node element, a minimal of six wave modes from three source points are required. It should be noted that if a six-node equilateral triangle element is formulated by using the spherical wave modes from the three source points lying in the lines which pass through the element center and parallel to its edges, the resulting element would be rank deficient. On the other hand, a rank sufficient element can be obtained by employing the wave modes from the three sources points which are located in the lines passing through the element center and its corner nodes, see Figure 3(b). These observations on the element rank are similar to those of the six-node hybridTrefftz plane element formulated with plane-wave modes [26]. To generalize the successful modes to a general triangular element, two methods of selecting the spherical wave modes are employed.

\subsection{Node-Centre Modes}

The spherical wave modes arising from the first method will be termed as "node-centre" modes. In this method, the parametric centre 0 with following coordinates given by (6):

$$
r_{0}=\left.\sum_{i=1}^{6} N_{i}\right|_{t=s=1 / 3} r_{i}, \quad z_{0}=\left.\sum_{i=1}^{6} N_{i}\right|_{t=s=1 / 3} z_{i}
$$

will be employed. The source points $\mathrm{A}\left(0, z_{A}\right), \mathrm{B}\left(0, z_{B}\right)$ and $\mathrm{C}\left(0, z_{C}\right)$ are the $z$-intercepts of Q-1, Q-2 and Q-3 as shown in Figure 3(a). It is trivial that

$$
z_{A}=\frac{r_{1} z_{0}-r_{0} z_{1}}{r_{1}-r_{0}}, z_{B}=\frac{r_{2} z_{0}-r_{0} z_{2}}{r_{2}-r_{0}}, z_{C}=\frac{r_{3} z_{0}-r_{0} z_{3}}{r_{3}-r_{0}} .
$$

The treatment for the cases that a $z$-intercept is far away from the element will be discussed in Section 4.3. The element employing the above source points and thus the following shape function matrix for the domain approximation: 


$$
\mathbf{P}=\left[\frac{R_{A 0}}{R_{A}}\left[\cos \left(k R_{A}\right), \sin \left(k R_{A}\right)\right], \frac{R_{B 0}}{R_{B}}\left[\cos \left(k R_{B}\right), \sin \left(k R_{B}\right)\right], \frac{R_{C 0}}{R_{C}}\left[\cos \left(k R_{C}\right), \sin \left(k R_{C}\right)\right]\right]
$$

will be termed as SATN. In the above matrix,

$$
R_{A}=\sqrt{r^{2}+\left(z-z_{A}\right)^{2}}, R_{B}=\sqrt{r^{2}+\left(z-z_{B}\right)^{2}} \text { and } R_{C}=\sqrt{r^{2}+\left(z-z_{C}\right)^{2}}
$$

whilst the normalization factors $R_{A 0}, R_{B 0}$ and $R_{C 0}$ are respectively the values of $R_{A}, R_{B}$ and $R_{C}$ at $0\left(r_{0}, z_{0}\right)$.

The $z$-intercepts of $0-4,0-5$ and $0-6$ constitute another set of source points. With them, the resulting element will be termed as SATN*.

\subsection{Equal-spaced Modes}

The spherical wave modes arising from the second method will be termed as "equal-spaced" modes. In this method, source points $\mathrm{Q}\left(r_{Q}, z_{Q}\right)$ 's are the Fermat points (also known as Torricelli points and the 1st isogonic centers) of the triangles formed by the corner or side nodes [28]. Taking the Fermat point of nodes $(1,2,3)$ as the illustration, Q-1, Q-2 and Q-3 are equal-spaced as shown in Figure 3(b). The condition can be mathematically expressed as:

$$
\frac{\left(z_{1}-z_{Q}\right)\left(r_{2}-r_{Q}\right)-\left(r_{1}-r_{Q}\right)\left(z_{2}-z_{Q}\right)}{\left(r_{1}-r_{Q}\right)\left(r_{2}-r_{Q}\right)+\left(z_{1}-z_{Q}\right)\left(z_{2}-z_{Q}\right)}=\sqrt{3}, \frac{\left(z_{2}-z_{Q}\right)\left(r_{3}-r_{Q}\right)-\left(r_{2}-r_{Q}\right)\left(z_{3}-z_{Q}\right)}{\left(r_{2}-r_{Q}\right)\left(r_{3}-r_{Q}\right)+\left(z_{2}-z_{Q}\right)\left(z_{3}-z_{Q}\right)}=\sqrt{3} .
$$

By solving them,

$$
\begin{aligned}
& r_{Q}=\frac{1}{W} \sum_{i=1}^{3}\left(\frac{\left(r_{i}^{2}-3 z_{i}^{2}\right)\left(z_{j}-z_{k}\right)-4 r_{i} z_{i}\left(r_{j}-r_{k}\right)}{2 \sqrt{3}}+\frac{\left(r_{i}^{2}+z_{i}^{2}\right)\left(r_{j}+r_{k}\right)}{2}-r_{i} z_{j} z_{k}\right)-\frac{3 r_{1} r_{2} r_{3}}{W}, \\
& z_{Q}=\frac{1}{W} \sum_{i=1}^{3}\left(\frac{4 r_{i} z_{i}\left(z_{j}-z_{k}\right)-\left(z_{i}^{2}-3 r_{i}^{2}\right)\left(r_{j}-r_{k}\right)}{2 \sqrt{3}}+\frac{\left(r_{i}^{2}+z_{i}^{2}\right)\left(z_{j}+z_{k}\right)}{2}-z_{i} r_{j} r_{k}\right)-\frac{3 z_{1} z_{2} z_{3}}{W}
\end{aligned}
$$

where

$$
W=\sum_{i=1}^{3}\left(\frac{\left(r_{i}-r_{j}\right)^{2}+\left(z_{i}-z_{j}\right)^{2}}{2}+\sqrt{3} x_{i}\left(r_{j}-z_{k}\right)\right), j=\bmod (i, 3)+1 \text { and } k=\bmod (i+1,3)+1 .
$$

Now, the source points $\mathrm{A}\left(0, z_{A}\right), \mathrm{B}\left(0, z_{B}\right)$ and $\mathrm{C}\left(0, z_{C}\right)$ are the $z$-intercepts of Q-1, Q-2 and Q-3. Similar to (18),

$$
z_{A}=\frac{r_{1} z_{Q}-r_{Q} z_{1}}{r_{1}-r_{Q}}, z_{B}=\frac{r_{2} z_{Q}-r_{Q} z_{2}}{r_{2}-r_{Q}} \text { and } z_{C}=\frac{r_{3} z_{Q}-r_{Q} z_{3}}{r_{3}-r_{Q}}
$$

The six-node element employing the above source points and thus the following shape function matrix for the domain approximation: 


$$
\mathbf{P}=\left[\frac{R_{A Q}}{R_{A}}\left[\cos \left(k R_{A}\right), \sin \left(k R_{A}\right)\right], \frac{R_{B Q}}{R_{B}}\left[\cos \left(k R_{B}\right), \sin \left(k R_{B}\right)\right], \frac{R_{C Q}}{R_{C}}\left[\cos \left(k R_{C}\right), \sin \left(k R_{C}\right)\right]\right]
$$

will be termed as SATE. In the above matrix, $R_{A}, R_{B}$ and $R_{C}$ have been defined in (20) whilst the normalization factors $R_{A Q}, R_{B Q}$ and $R_{C Q}$ are respectively the values of $R_{A}, R_{B}$ and $R_{C}$ at $\mathrm{Q}\left(r_{Q}, z_{Q}\right)$.

By changing nodes $(1,2,3)$ to nodes $(4,5,6)$, another point $Q^{*}$ inside the element can be obtained such that $\mathrm{Q}^{*}-4, \mathrm{Q}^{*}-5$ and $\mathrm{Q} *_{-} 6$ can define another set of source points. The resultant element will be denoted as SATE*.

\subsection{Treatment for a Distant Source Point}

It may happen that a source point $S\left(0, z_{S}\right)$ is far away from the element within which the related wave is essentially parallel to the $z$-axis. To avoid numerical difficulty associated with the large radial distant $R_{S}$, the two related spherical wave modes will be replaced by the two plane wave modes along the $z$-axis:

$$
\left\{\cos \left(k\left(z-\left.z\right|_{s=t=1 / 3}\right)\right), \sin \left(k\left(z-\left.z\right|_{s=t=1 / 3}\right)\right)\right\} \quad \text { if } \quad\left|\frac{\left.r\right|_{s=t=1 / 3}}{\left.z\right|_{s=t=1 / 3}-z_{S}}\right|<\tan \chi
$$

where $\chi$ is the small angle and is here taken rather arbitrarily to be $\pi / 120$.

\subsection{Singularity at the Source Point}

The wave mode $\cos (k R) / R$ is singular at its source point where $R=0$. When the source point is too close to the element, the singularity may pose numerical difficulty. A simple remedy to avoid the problem if to replace the hybrid element with the conventional one when $r=0$ in one or more element nodes.

\section{NUMERICAL EXAMPLES}

In this section, the predictions of the afore-discussed axial symmetric triangular elements are reported. These elements include:

- CAT - the conventional six-node element, see Section 2.

- SATN - the six-node hybrid element based on the node-centre modes with their source points defined by 0-1, 0-2 and 0-3, see Section 4.1.

- SATE - the six-node hybrid element based on the equal-spaced modes with their source points defined by Q-1, Q-2 and Q-3, see Section 4.2. 
To enhance the graphical clarity in portraying the numerical predictions, SATN* discussed at the end of Section 4.1 will not be reported. SATN* element is slightly less accurate than SATN and its predictions are graphically indistinguishable from those of SATN. For the similar reasons, SATE* discussed at the end of Section 4.2 will not be reported.

Unless specified, all elements are evaluated by the six-point domain integration rule [30]. Higher order integration rules yield practicably identical results. To compare the accuracy of the elements, the normalized error and the relative error with respect to the error of the conventional element will be computed. They are

$$
\begin{aligned}
& \text { Normalized error }=\left(\frac{\int_{\Omega}\left(u-u^{\text {exact }}\right)^{2} d \Omega}{\int_{\Omega}\left(u^{\text {exact }}\right)^{2} d \Omega}\right)^{1 / 2}=\left(\frac{\sum_{e} \int_{\Omega^{e}}\left(u-u^{\text {exact }}\right)^{2} d \Omega}{\sum_{e} \int_{\Omega^{e}}\left(u^{\text {exact }}\right)^{2} d \Omega}\right)^{1 / 2}, \\
& \text { Relative error }=\left(\frac{\int_{\Omega}\left(u-u^{\text {exact }}\right)^{2} d \Omega}{\int_{\Omega}\left(u^{c}-u^{\text {exact }}\right)^{2} d \Omega}\right)^{1 / 2}=\left(\frac{\sum_{e} \int_{\Omega^{e}}\left(u-u^{\text {exact }}\right)^{2} d \Omega}{\sum_{e} \int_{\Omega^{e}}\left(u^{c}-u^{\text {exact }}\right)^{2} d \Omega}\right)^{1 / 2}
\end{aligned}
$$

where $u$ denotes the finite element prediction, $u^{\text {exact }}$ denotes the exact solution, $u^{c}$ denotes the prediction of the conventional element CAT. In the hybrid elements, $g$ is the prediction within the element domain and, thus, $u$ is replaced by $g$. In both error measures, the area integrals are evaluated by the six-point domain integration. Again, higher order integration rules yield practicably identical results. In the examples, the number of nodal spacings per wavelength

$$
N_{n}=\text { wave length } / \text { nodal spacing }=2 \pi /(k h)
$$

will be specified. In the expression, $h$ denotes the nodal spacing. For conventional element models, many literatures recommend $N_{n}>10$ (see, e.g., [21,29] among others).

\subsection{Condition Number versus Integration Order and Invariance}

The element in Figure 2(b) is considered. Nodes H and J bisect EF and EG, respectively. The element edge lengths are $1, \sqrt{ } 0.97$ and $\sqrt{ } 1.17$ which gives an average nodal spacing of 0.5 approximately. Two wavenumbers $k=1.25$ and 2.5 are considered. The relevant $N_{n} \mathrm{~s}$ are approximately equal to 10 and 5, respectively. Let $|\alpha|$ denote the eigenvalue magnitude of the element matrix, $|\alpha|_{\max },|\alpha|_{\min }$ and the condition number $N_{c}\left(=|\alpha|_{\max } /|\alpha|_{\min }\right)$ are computed. Table 1 lists the computed values of the elements when the element matrices are evaluated by three-point, six- 
point and seven-point domain integration rules [30]. There are two three-point rules and only the more popular one, in which all the sampling points are away from the element boundary, is considered. It can be seen that $N_{c}$ changes considerably when the integration changes from threepoint to six-point rule. Numerical tests also indicate that the elements evaluated by the six-point rule are markedly more accurate than those by the three-point rule.

Different nodal connectivity orders which define the directions of the parametric axes are also attempted. The elements are also shifted along the $z$-direction. The computed values do not vary with the connectivity and the rigid body translation. Thus, the elements are invariant $[31,32]$.

Table 1. $|\alpha|_{\max },|\alpha|_{\min }$ and $N_{c}$ of the elements under different domain integration rules.

\begin{tabular}{|c|c|c|c|c|c|c|c|c|c|c|}
\hline \multicolumn{2}{|c|}{} & \multicolumn{3}{|c|}{ three-point rule } & \multicolumn{3}{c|}{ six-point rule } & \multicolumn{3}{c|}{ seven-point rule } \\
\cline { 2 - 11 } \multicolumn{2}{|c|}{} & $|\alpha|_{\max }$ & $|\alpha|_{\min }$ & $N_{c}$ & $|\alpha|_{\max }$ & $|\alpha|_{\min }$ & $N_{c}$ & $|\alpha|_{\max }$ & $|\alpha|_{\min }$ & $N_{c}$ \\
\hline \multirow{2}{*}{ CAT } & $k=1.25$ & 27.75 & 1.349 & 20.57 & 27.45 & 1.476 & 18.60 & 27.46 & 1.476 & 18.61 \\
\cline { 2 - 11 } & $k=2.5$ & 27.00 & 1.979 & 13.64 & 25.60 & 2.134 & 11.99 & 25.65 & 2.129 & 12.05 \\
\hline \multirow{2}{*}{ SATN } & $k=1.25$ & 27.52 & 1.474 & 18.68 & 26.17 & 1.481 & 17.67 & 26.21 & 1.481 & 17.69 \\
\cline { 2 - 11 } & $k=2.5$ & 26.89 & 1.992 & 13.50 & 24.41 & 2.066 & 11.81 & 24.53 & 2.061 & 11.90 \\
\hline \multirow{2}{*}{ SATE } & $k=1.25$ & 27.60 & 1.474 & 18.73 & 26.36 & 1.481 & 17.80 & 26.39 & 1.481 & 17.82 \\
\cline { 2 - 10 } & $k=2.5$ & 26.95 & 1.991 & 13.53 & 24.56 & 2.070 & 11.86 & 24.68 & 2.064 & 11.96 \\
\hline
\end{tabular}

\subsection{Plane-Wave Problem}

Figure 4 depicts a square and rhombic annulars with centers at $(L, 0)$ and sizes $L \times L$ where $L=2$. The domain boundaries are prescribed with the boundary conditions derived from the following plane wave solution:

$$
u=\cos (k z)
$$

The typical mesh layout can also be seen in Figure 4. With $k L$ fixed to either 10 or 20, the mesh density is varied such that $N_{n}$ ranges from $2 \pi$ to $8 \pi$.

Square Annular - Figures 5(a) and 5(b) plot the normalized errors against the total number of nodes employed in modelling the square annular which is prescribed with the natural boundary condition. It can be seen that the convergence rates of the compared elements are essentially the same when the mesh is dense. Figure 5(a) shows the results for $k L=10$. At the highest mesh density, the relative errors of SATN and SATE are $21.5 \%$ and $33.7 \%$, respectively. Figure 5(b) shows the results for $k L=20$. At the highest mesh density, the relative errors of SATN and SATE 
with respect to CAT are $57.6 \%$ and $52.2 \%$, respectively.

Rhombic Annular - Figures 6(a) and 6(b) plot the normalized errors against the total number of nodes employed in modelling the rhombic annular which is prescribed with the essential boundary condition. Again, the convergence rates of the compared elements are essentially the same when the mesh is dense. Figure 6(a) shows the results for $k L=10$. At the highest mesh density, the relative errors of SATN and SATE are both $42.0 \%$. Figure 6(b) shows the results for $k L=20$. At the highest mesh density, the relative errors of SATN and SATE are both $48.9 \%$.

\subsection{Cylindrical-Wave Problem}

In this section, a cylindrical-wave problem is studied by using the same domains and meshes as the last subsection, see Figure 4. Both domain boundaries are prescribed with the boundary conditions derived from the following cylindrical wave solution:

$$
u=J_{0}(k r)
$$

where $J_{0}$ is the zeroth order Bessel function of the first kind. Now, $k L$ is fixed to either 16 or 32 whereas the mesh density is varied such that $N_{n}$ ranges from $2 \pi$ to $8 \pi$.

Square Annular - Figures 7(a) and 7(b) plot the normalized errors against the total number of nodes employed in modelling the square annular which is prescribed with the natural boundary condition. Again, the convergence rates of the compared elements are essentially the same when the mesh is dense. Figure 7(a) shows the results for $k L=16$. At the highest mesh density, the relative errors of SATN and SATE are $28.3 \%$ and $36.0 \%$, respectively. Figure 7(b) shows the results for $k L$ $=32$. At the highest mesh density, the relative errors of SATN and SATE with respect to CAT are $32.2 \%$ and 29.7 , respectively.

Rhombic Annular - Figures 8(a) and 8(b) plot the normalized errors against the total number of nodes employed in modelling the rhombic annular which is prescribed with the essential boundary condition. Figure 8(a) shows the results for $k L=16$. At the highest mesh density, the relative errors of SATN and SATE are $60.5 \%$ and $71.6 \%$, respectively. Figure 8(b) shows the results for $k L=32$. At the highest mesh density, the relative errors of SATN and SATE are $61.6 \%$ and $71.2 \%$, respectively.

\subsection{Spherical-wave Problem}

Figure 9 represents a typical mesh in which the number of elements along each coordinate axis is 8 and the number of elements is 96 for a hemispherical domain with radius $R=1$. Boundary conditions corresponding to the following spherical wave solution: 


$$
u=\frac{\sin \left(k \sqrt{r^{2}+z^{2}}\right)}{k \sqrt{r^{2}+z^{2}}}
$$

are implemented. The conditions include $u=1$ at $(0,0), u,_{r}=0$ along $r=0, u,{ }_{z}=0$ along $z=0$ and $u$ $=\sin (k R) /(k R)$ on $r^{2}+z^{2}=R^{2}$. With $k R$ fixed to either 10 or 20 , the mesh density is varied such that $N_{n}$ ranges from $2.4 \pi$ to $9.6 \pi$. Figures 10 (a) and 10 (b) plot the normalized errors against the total number of nodes employed in modelling the hemisphere. Same as the last examples, the convergence rates of the compared elements are essentially the same when the mesh is dense. Figure 10(a) shows the results for $k R=10$. At the highest mesh density, the relative errors of SATN and SATE are 70.1 $\%$ and $56.5 \%$, respectively. Figure $10(\mathrm{~b})$ shows the results for $k R=20$. At the highest mesh density, the relative errors of SATN and SATE are $67.6 \%$ and $56.8 \%$, respectively.

\section{CONCLUSION}

Six-node hybrid-Trefftz triangular axial symmetric elements are devised in this paper. They have the same boundary degrees of freedom as the conventional element, which is based on a single field variational functional, and can be readily be incorporated into the standard finite element program framework. Boundary conditions can also be prescribed as if they were conventional elements. A hybrid variational functional that enforces the equality of the two fields inter alia is employed. While the boundary field is approximated by the standard nodal interpolation, the domain field satisfies the Helmholtz equation and is composed of spherical waves with source points located along the axis of symmetry. Two ways of selecting the source points have been attempted and they lead to the "nodecentre" and "equal-spaced" modes. For the attempted examples, the average error ratios of the proposed elements and the conventional element are marginally less than $50 \%$ at considerably dense meshes. On the other hand, the convergent rate of the proposed and conventional elements are essentially the same. This is well-expected as the convergent rate is limited by the orders of boundary and domain approximations whichever the lower.

Acknowledgment - The support of Hong Kong Research Grant Council in the form of a GRF grant (HKU 7167 08E) is gratefully acknowledged. 


\section{REFERENCES}

[1] Pian THH. Derivation of element stiffness matrices by assumed stress distribution. J.AIAA 2: 1332-1336 (1964)

[2] Tong P, Pian THH, Lasry SL. A hybrid-element approach to crack problems in plane elasticity. Int.J.Numer.Meth.Engng. 7: 297-308 (1973)

[3] Atluri SN, Kobayashi AS, Nakagaki M. An assumed displacement hybrid finite element method for fracture mechanics. Int.J.Fracture 11: 257-271 (1975)

[4] Pian THH. State-of-the-art development of hybrid/mixed finite element method. Finite Elements in Analysis \& Design 21: 5-20 (1995)

[5] Sze KY, Chan WK, Pian THH. An eight-node hybrid-stress solid-shell element for geometric nonlinear analysis of elastic shells. Int.J.Numer.Meth.Engng. 55: 853-878 (2000)

[6] Jirousek J. Basis for development of large finite elements locally satisfying all field equations. Comput.Meth. Appl.Mech.Engng. 14: 65-92 (1978)

[7] Jirousek J, Zieliński AP. Survey of Trefftz-type element formulations. Computers \& Structures 63: 225-242 (1997)

[8] Freitas JA, Almeida JP, Pereira EMB. Non-conventional formulations for the finite element method. Comput.Mech. 23: 488-501 (1999)

[9] Chen MC, Sze KY, Wang HT. Analysis of singular stresses in bonded bimaterial wedges by computed eigen solutions and hybrid element method. Commun.Numer.Methods Engrg. 17: 495-507 (2001)

[10] Wang HT, Sze KY, Yang XM. Analysis of electromechanical stress singularity in piezoelectrics by computed eigensolutions and hybrid-trefftz finite element models. Comput. Mech. 38: 551564 (2006)

[11] Zielinski AP, Herrera I. Trefftz method: fitting boundary conditions. Int.J.Numer.Meth.Engng. 24: 871-891 (1987)

[12] Cheung YK, Jin WG, Zienkiewicz OC. Solution of Helmholtz equation by Trefftz method. Int.J.Numer. Meth.Engng. 32 63-78 (1991)

[13] Herrera I. Trefftz method: A general theory. Numerical Meth.for Partial Differential Equations 16: 561-580 (2000)

[14] Sladek J, Sladek V, Keer RV. Global and local Trefftz boundary integral formulations for sound vibration. Advances in Engineering Software 33: 469-476 (2002)

[15] Chang JR, Liu RF. An asymmetric indirect Trefftz method for solving free-vibration problems. $J$. Sound \& Vibration 275: 991-1008 (2004)

[16] Stojek M. Least square Trefftz-type elements for the Helmholtz equation. Int.J.Numer.Meth. Engng. 41: 831-849 (1998)

[17] Stojek M, Markiewicz M, Mahrenholtz O. Diffraction loads on multiple vertical cylinders with rectangular cross section by Trefftz-type finite elements, Computers \& Structures 75: 335-345 (2000)

[18] Freitas JAT, Cismaşiu C. Hybrid-Trefftz displacement element for spectral analysis of bounded and unbounded media. Int.J.Solids \& Structures 40: 671-699 (2003)

[19] Melenk JM, Babuška I. The partition of unity method. Int.J.Numer.Meth.Engng. 40:727- 758 (1997)

[20] Mayer P, Mandel J. The finite ray element method for the Helmholtz equation of scattering: first numerical experiments. Report 111, Center of Computational Mathematics, University of Colorado at Denver, 1997.

[21] Laghrouche O, Bettess P. Short wave modelling using special finite elements. J. Computational Acoustics 8: 189-210 (2000).

[22] Ortiz P, Sanchez E. An improved partition of unity finite element method for diffraction problems. Int.J.Numer.Meth.Engng. 50:2727- 2740 (2001). 
[23] Farhat C, Harari I, Hetmaniuk U. The discontinuous enrichment method for multiscale analysis. Computer Meth.in Appl.Mech. \& Engng. 192: 1389-1419 (2003)

[24] Farhat C, Harari I, Hetmaniuk U. A discontinuous Galerkin method with Lagrange multipliers for the solution of Helmholtz problems in the mid-frequency regime. Computer Meth.in Appl.Mech. \& Engng. 192: 3195-3209 (2003)

[25] Sze KY, Cheung YK. A hybrid-Trefftz finite element model for Helmholtz problem. Comm. Numer.Meth.Engng. 24: 2047-2060 (2008)

[26] Sze KY, Liu GH. Hybrid-Trefftz six-node triangular finite element models for Helmholtz problem, Computational Mechanics 46: 455-470 (2010)

[27] Sze KY, Liu GH, Fan H. Four- and eight-node hybrid-Trefftz quadrilateral finite element models. Computer Methods Applied Mechanics \& Engrg. 199: 598-614 (2010)

[28] Kimberling C. Triangle centers and central triangles. Congr.Numer. 129:1-295 (1998)

[29] Deraemaeker A, Babuška I, Bouillard P. Dispersion and pollution of the fem solution for the Helmholtz equation in one, two and three dimensions. Inter.J.Numer.Meth.Engng. 46: 471499 (1999).

[30] Cook RD. Malkus DS, Plesha ME. Concepts and Applications of Finite Element Analysis. Third Edn., John Wiley \& Sons, 1989.

[31] Spilker RL, Maskeri SM, Kania E. Plane isoparametric hybrid-stress elements: invariance and optimal sampling. Int.J.Numer.Meth.Engng., 17, 1469-1496 (1981)

[32] Sze KY, Chow CL, Chen WJ. On invariance of isoparametric hybrid elements. Commun. Numer.Meth.Engng. 8: 385-406 (1992) 


\section{Appendix: Mathematical Preliminary for Axial Symmetry}

Consider the following general 3D position vector $\mathbf{R}$ and its differential $d \mathbf{R}$ :

$$
\mathbf{R}=x \mathbf{e}_{x}+y \mathbf{e}_{y}+z \mathbf{e}_{z}=\left\{\begin{array}{l}
x \\
y \\
z
\end{array}\right\}=\left\{\begin{array}{c}
r \cos \theta \\
r \sin \theta \\
z
\end{array}\right\}, d \mathbf{R}=\left\{\begin{array}{c}
\cos \theta \\
\sin \theta \\
0
\end{array}\right\} d r+r\left\{\begin{array}{c}
-\sin \theta \\
\cos \theta \\
0
\end{array}\right\} d \theta+\left\{\begin{array}{l}
0 \\
0 \\
1
\end{array}\right\} d z
$$

where $r^{2}=x^{2}+y^{2}$ and $\tan \theta=y / x$. The covariant tensors $\boldsymbol{g}_{i}$ s and the contravariant tensors $\boldsymbol{g}^{i}$ s for the cylindrical coordinates $(r, \theta, z)$ can be derived as:

$$
\left[\mathbf{g}_{r}, \mathbf{g}_{\theta}, \mathbf{g}_{z}\right]=\left[\begin{array}{ccc}
\cos \theta & -r \sin \theta & 0 \\
\sin \theta & r \cos \theta & 0 \\
0 & 0 & 1
\end{array}\right], \quad\left[\mathbf{g}^{r}, \mathbf{g}^{\theta}, \mathbf{g}^{z}\right]=\left[\mathbf{g}_{r}, \mathbf{g}_{\theta}, \mathbf{g}_{z}\right]^{-T}=\left[\begin{array}{ccc}
\cos \theta & -(\sin \theta) / r & 0 \\
\sin \theta & (\cos \theta) / r & 0 \\
0 & 0 & 1
\end{array}\right] .
$$

The gradient operator $\nabla$ and Laplace operator $\nabla^{2}$ for the cylindrical coordinates are:

$$
\begin{gathered}
\nabla=\mathbf{g}^{r} \frac{\partial}{\partial r}+\mathbf{g}^{\theta} \frac{\partial}{\partial \theta}+\mathbf{g}^{z} \frac{\partial}{\partial z} \text { and } \\
\nabla^{2}=\left(\mathbf{g}^{r} \frac{\partial}{\partial r}+\mathbf{g}^{\theta} \frac{\partial}{\partial \theta}+\mathbf{g}^{z} \frac{\partial}{\partial z}\right)\left(\mathbf{g}^{r} \frac{\partial}{\partial r}+\mathbf{g}^{\theta} \frac{\partial}{\partial \theta}+\mathbf{g}^{z} \frac{\partial}{\partial z}\right)=\frac{1}{r} \frac{\partial}{\partial r}\left(r \frac{\partial}{\partial r}\right)+\frac{1}{r^{2}} \frac{\partial^{2}}{\partial \theta^{2}}+\frac{\partial^{2}}{\partial z^{2}}
\end{gathered}
$$

For any smooth functions $f=f(r, z)$ and $h=h(r, z)$,

$$
(\nabla f)^{T}(\nabla h)=\left(\mathbf{g}^{r} \frac{\partial f}{\partial r}+\mathbf{g}^{z} \frac{\partial f}{\partial z}\right)^{T}\left(\mathbf{g}^{r} \frac{\partial h}{\partial r}+\mathbf{g}^{z} \frac{\partial h}{\partial z}\right)=\frac{\partial f}{\partial r} \frac{\partial h}{\partial r}+\frac{\partial f}{\partial z} \frac{\partial h}{\partial z}, \nabla^{2} f=\frac{1}{r} \frac{\partial}{\partial r}\left(r \frac{\partial f}{\partial r}\right)+\frac{\partial^{2} f}{\partial z^{2}}
$$

and

$$
(\nabla h)^{T}(\nabla f)+h \nabla^{2} f=h\left[\frac{1}{r} \frac{\partial}{\partial r}\left(r \frac{\partial f}{\partial r}\right)+\frac{\partial^{2} f}{\partial z^{2}}\right]+\frac{\partial h}{\partial r} \frac{\partial f}{\partial r}+\frac{\partial h}{\partial z} \frac{\partial f}{\partial z}
$$

For any area $A$ in the $r$-z-plane, (33) and the Green's theorem yield

$$
\iint_{A}\left[(\nabla h)^{T}(\nabla f)+h \nabla^{2} f\right] r d r d z=\iint_{A}\left[\frac{\partial}{\partial r}\left(h r \frac{\partial f}{\partial r}\right)+\frac{\partial}{\partial z}\left(h r \frac{\partial f}{\partial z}\right)\right] d r d z=\oint_{\partial A} h\left(\cos \theta_{n} \frac{\partial f}{\partial r}+\sin \theta_{n} \frac{\partial f}{\partial z}\right) r d \Gamma
$$

Where $\partial A$ denotes the entire boundary of $A$ and $\theta_{n}$ is the inclination of the outward normal vector of the boundary to the $r$-axis, see Figure 1 . 


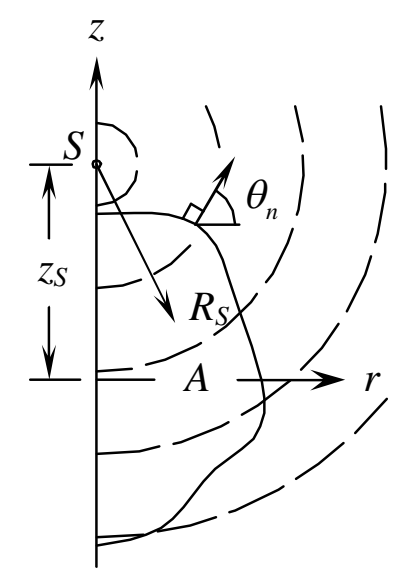

Figure 1. Cross section $A$ of an axial symmetric body in the $r$-z-plane. S denotes the source point of the spherical wave $u=\exp \left(i k R_{S}\right) /\left(k R_{S}\right)$ where $R_{S}^{2}=r^{2}+\left(z-z_{S}\right)^{2}$. Over the boundary of $A, \theta_{n}$ denotes the inclination of the outward normal vector to the $r$-axis.

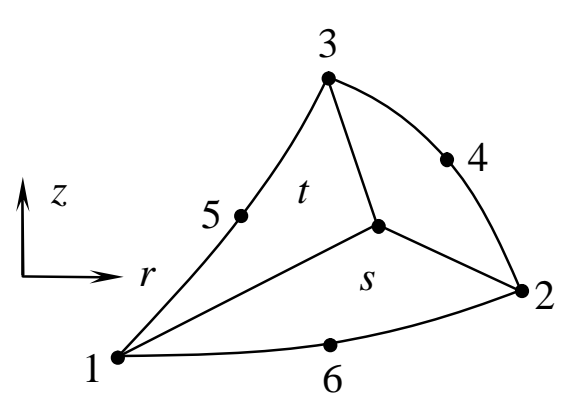

(a)

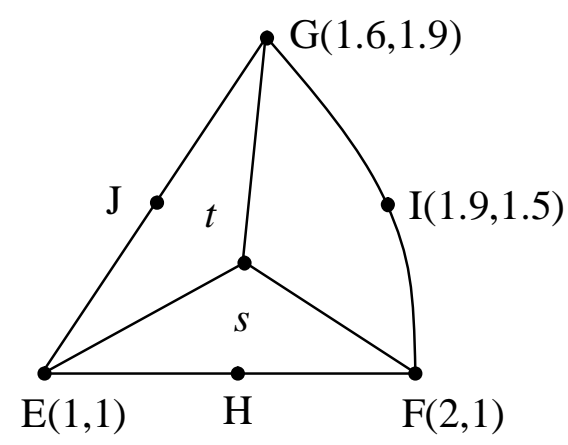

(b)

Figure 2. The six-node triangular element. (a) $s$ and $t \in[0,1]$ are the area coordinates. (b) The single for examining the condition numbers and invariance of the element matrices. 


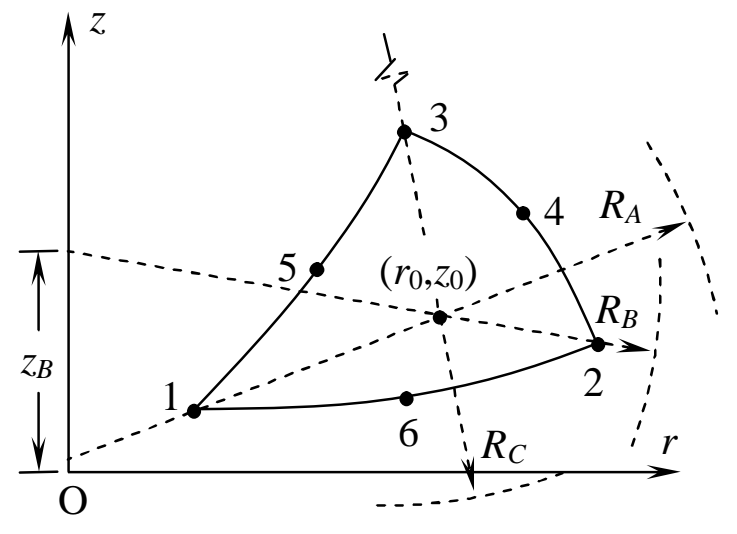

(a)

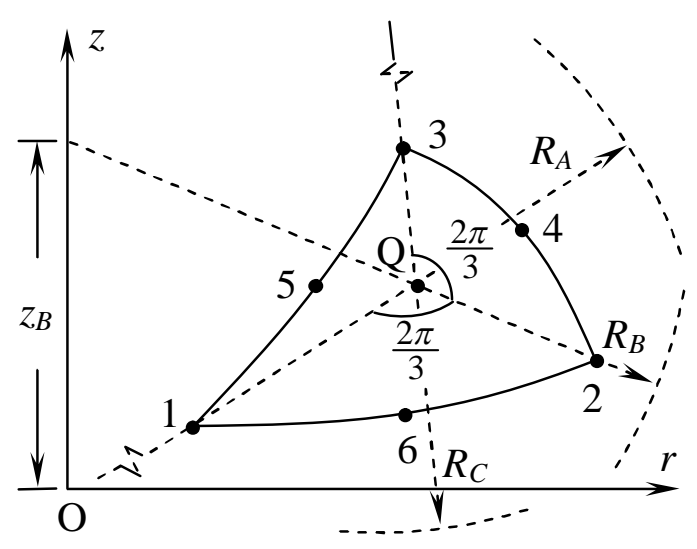

(b)

Figure 3. Directions of the spherical-wave modes employed in (a) SATN and (b) SATE. $0\left(r_{0}, z_{0}\right)$ with $\left(r_{0}, z_{0}\right)=\left.(r, z)\right|_{s=t=1 / 3}$ and $\mathrm{Q}\left(r_{Q}, z_{Q}\right)$ are points inside the element for locating the source points.

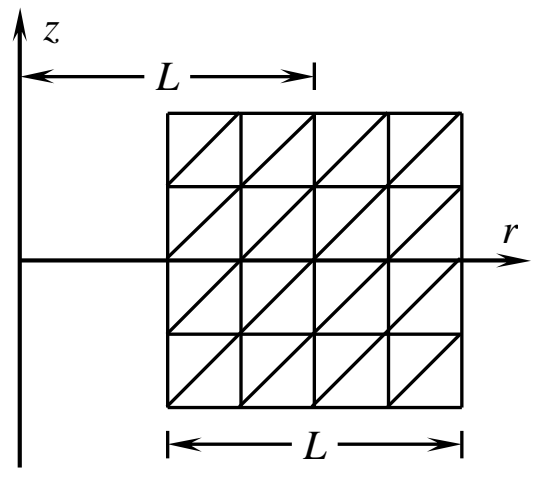

(a)

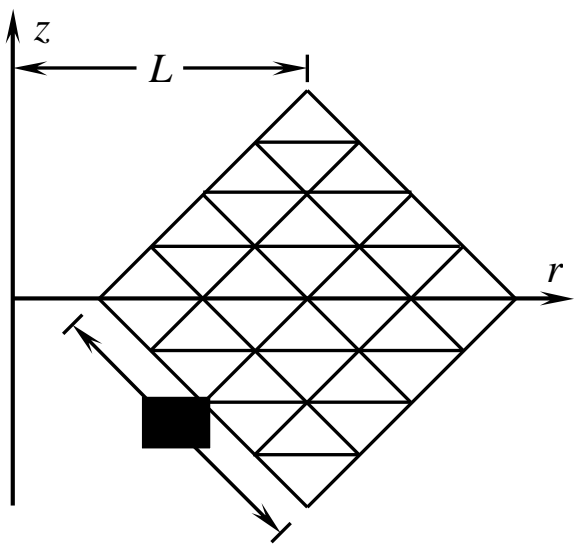

(b)

Figure 4. The (a) square and (b) rhombic annular domains with centres $(L, 0)$ and sizes $L \times L$ where $L$ $=2$. Both domains are meshed into $2 \times(4 \times 4)$ elements. Boundary conditions derived from the plane-wave and cylindrical-wave solutions are prescribed. 


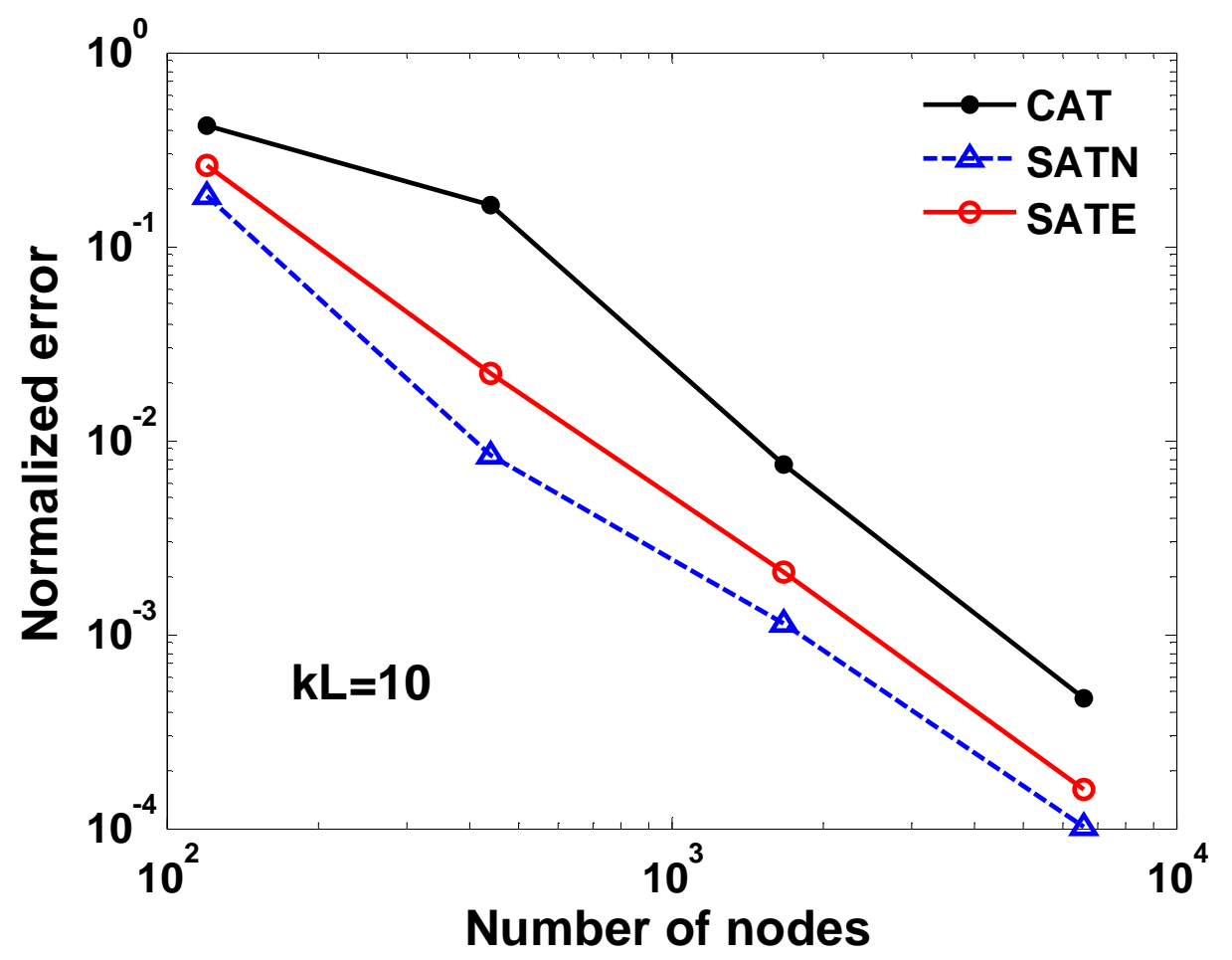

$5(\mathrm{a})$

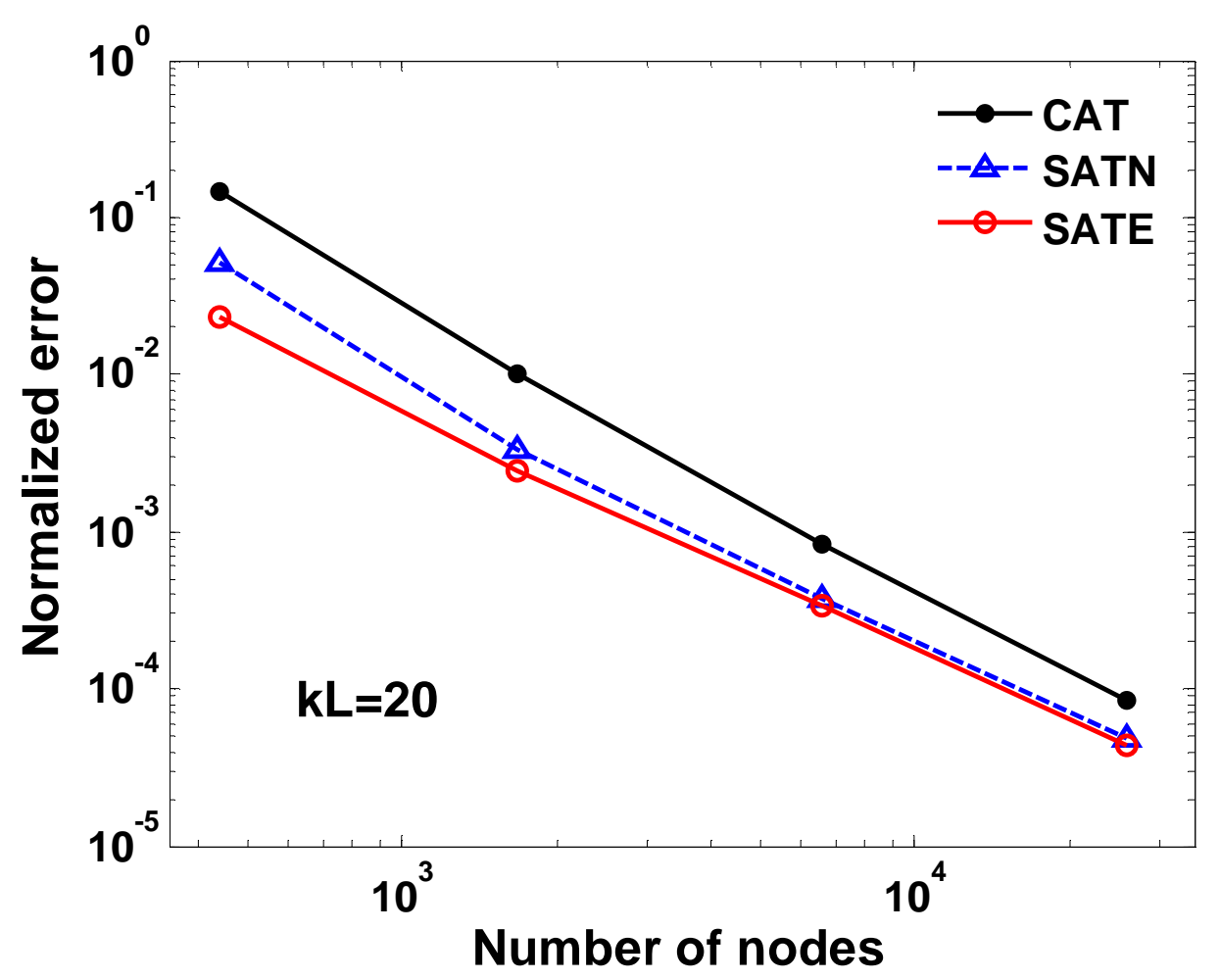

$5(b)$

Figure 5. Errors of the elements in the plane-wave problem considered by a square annular domain with kL (a) 10 and (b) 20 . The mesh density is varied such that $N_{n}$ ranges from $2 \pi$ to $8 \pi$. 


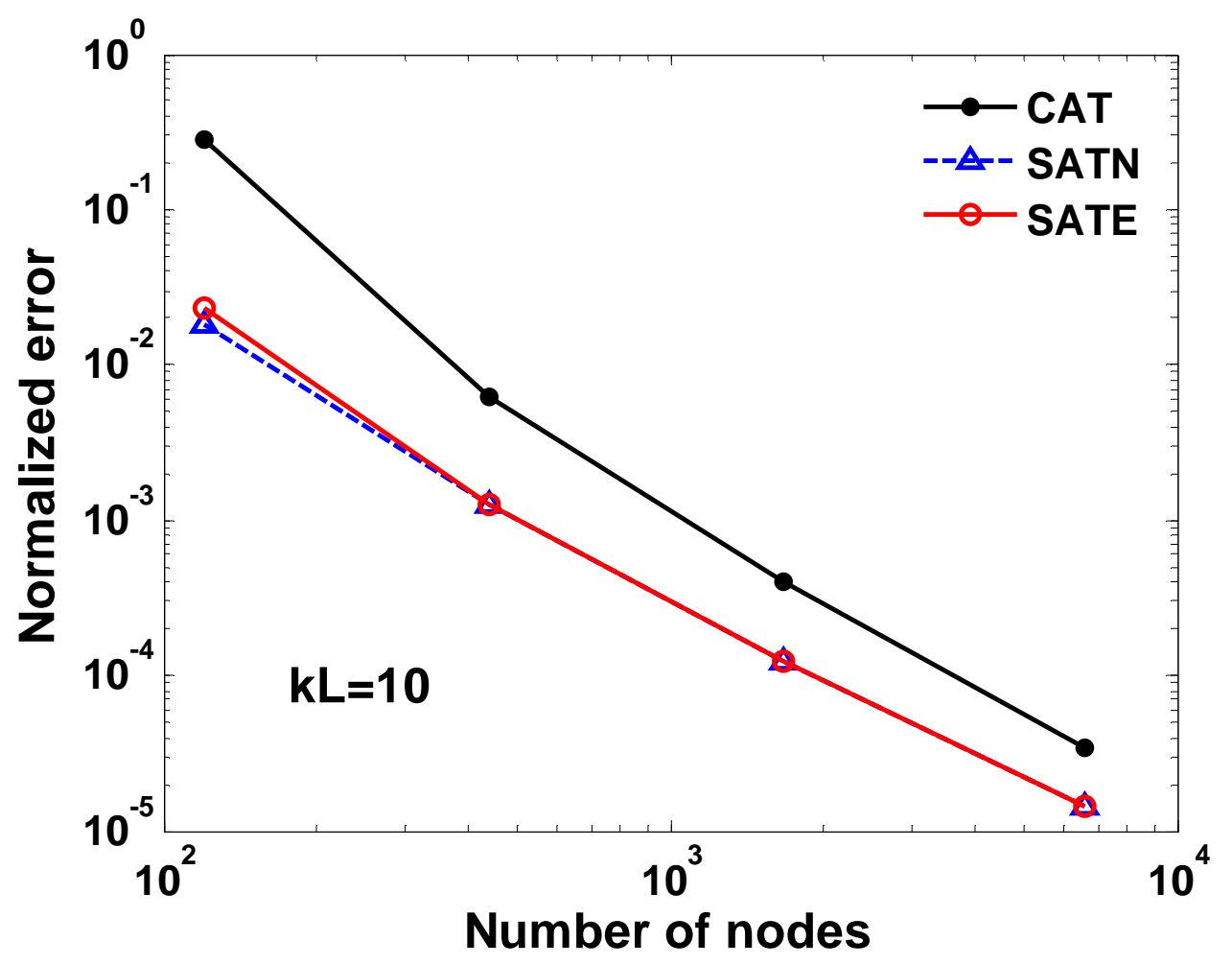

6(a)

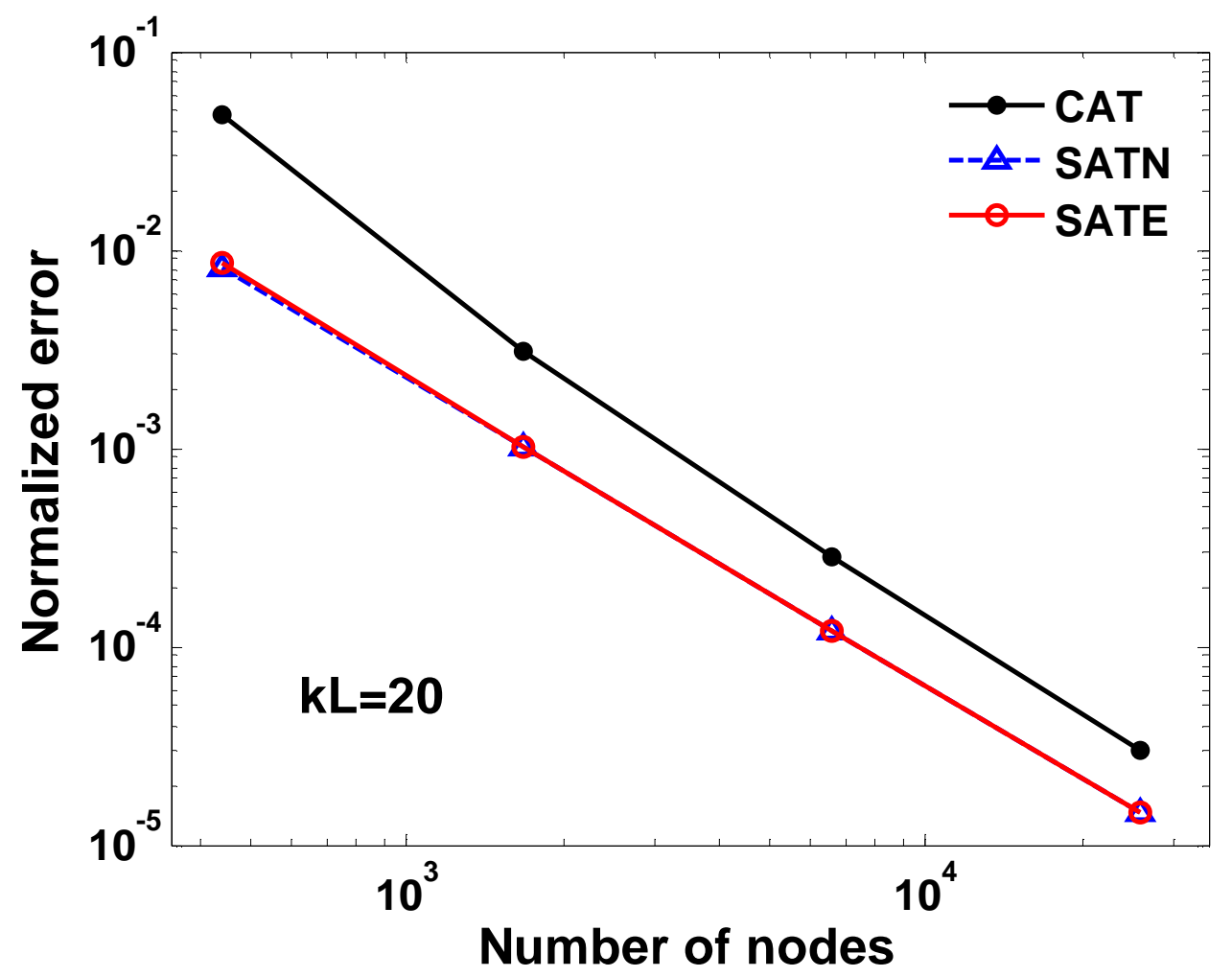

6(b)

Figure 6. Errors of the elements in the plane-wave problem considered by a rhombic annular domain with $k L=$ (a) 10 and (b) 20 . The mesh density is varied such that $N_{n}$ ranges from $2 \pi$ to $8 \pi$. 


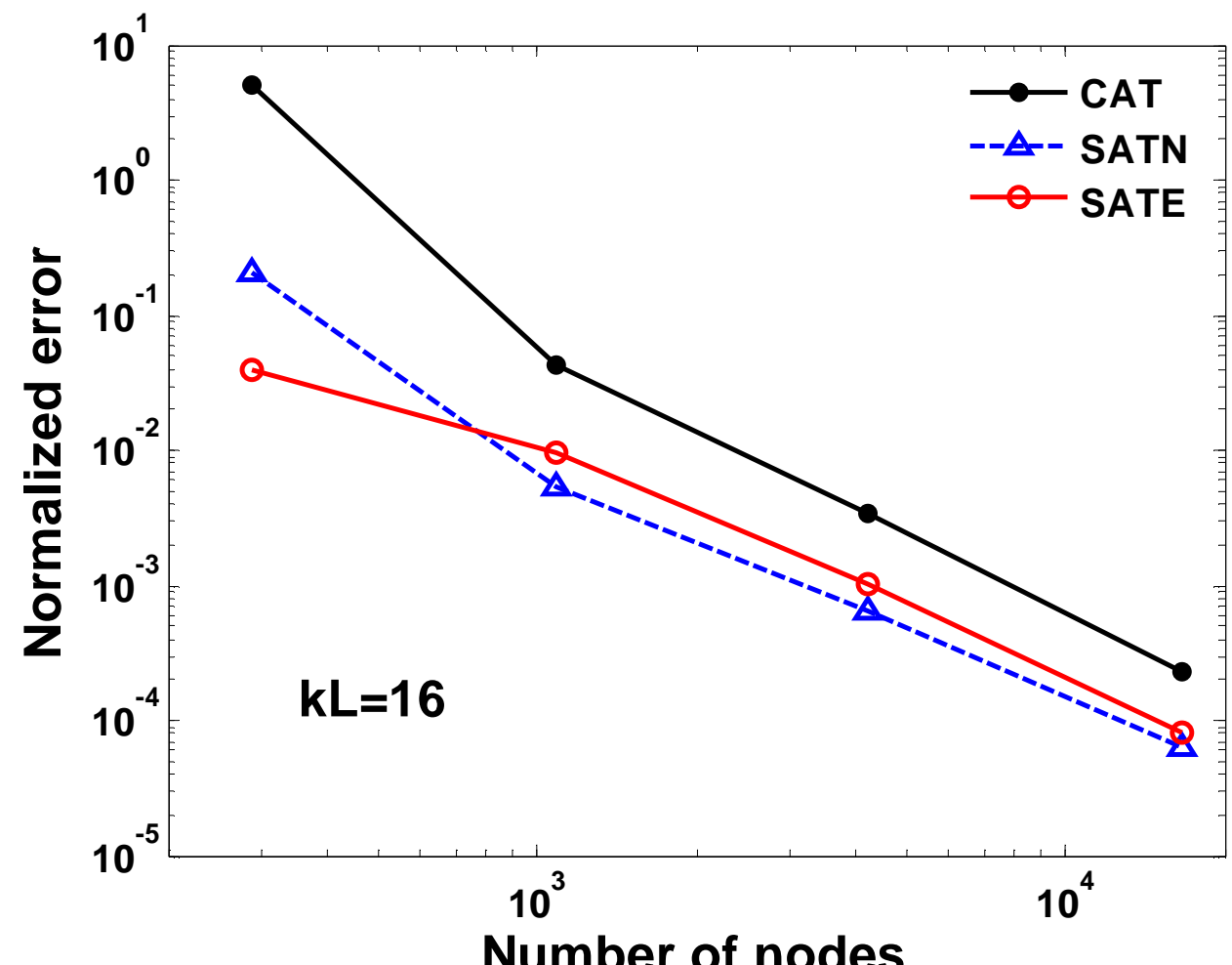

$7(\mathrm{a})$

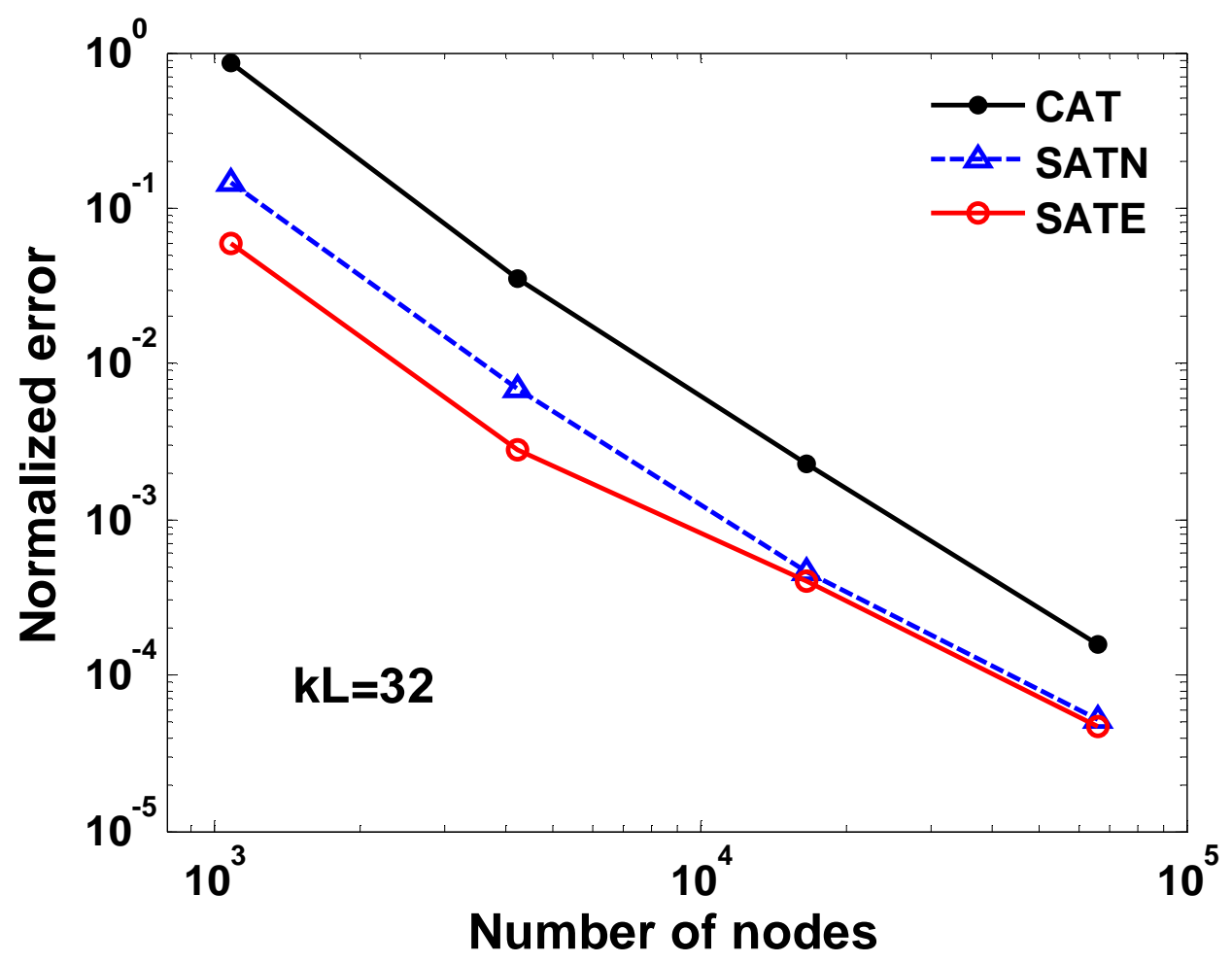

7(b)

Figure 7. Errors of the elements in the cylindrical-wave problem considered by a square annular domain with $k L=$ (a) 16 and (b) 32 . The mesh density is varied such that $N_{n}$ ranges from $2 \pi$ to $8 \pi$. 


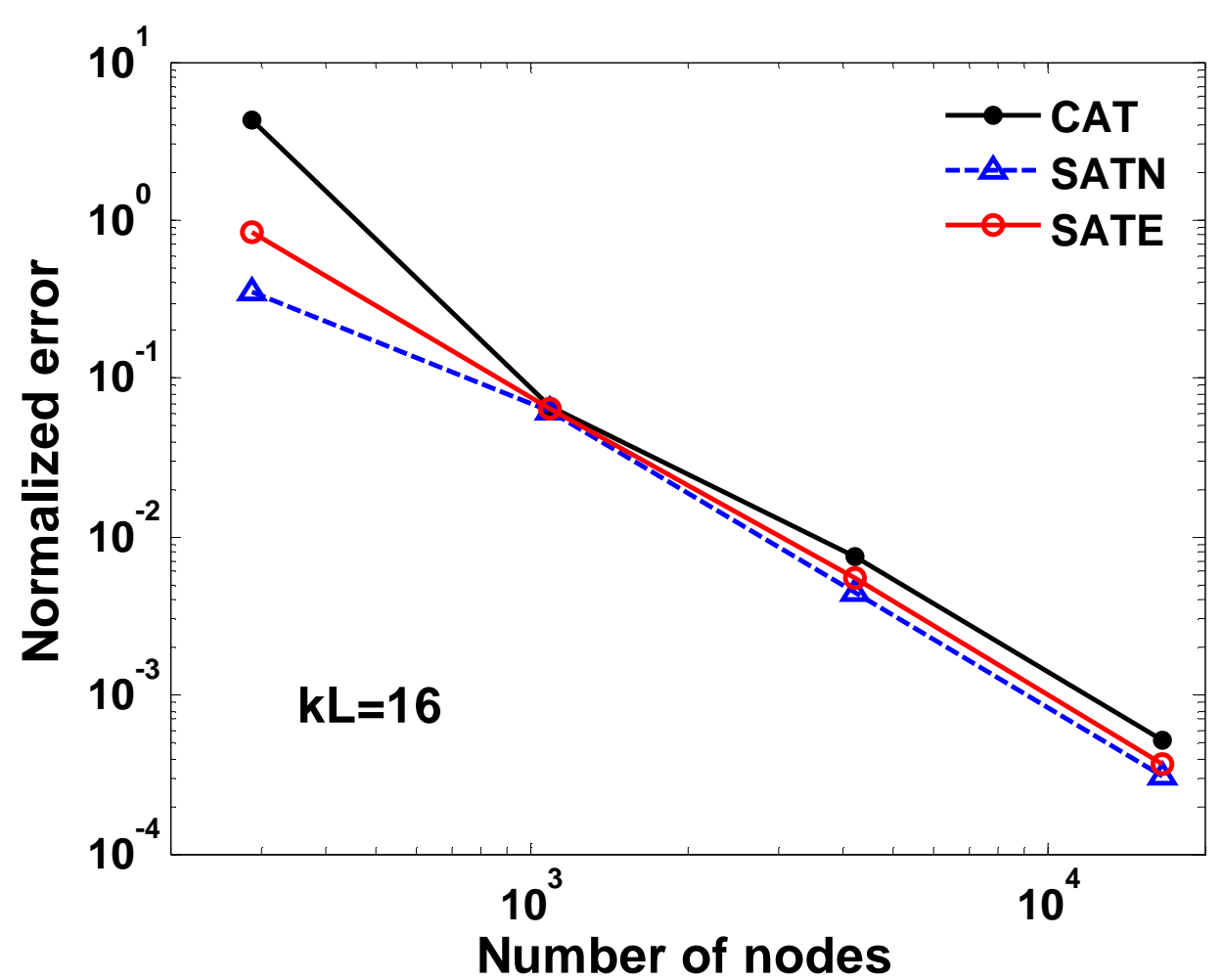

8(a)

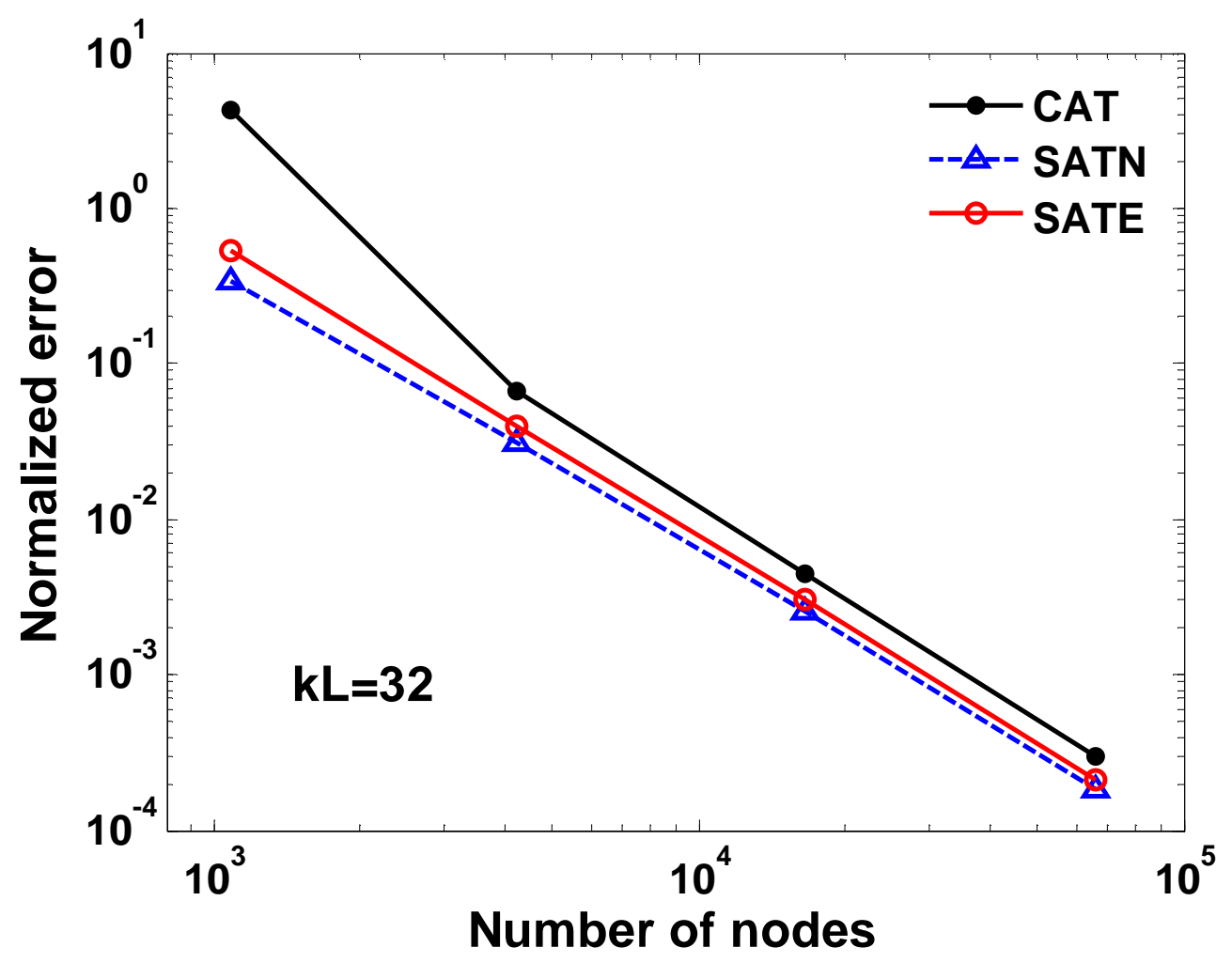

8(b)

Figure 8. Errors of the elements in the cylindrical-wave problem considered by a rhombic annular domain with $k L=$ (a) 16 and (b) 32. The mesh density is varied such that $N_{n}$ ranges from $2 \pi$ to $8 \pi$. 


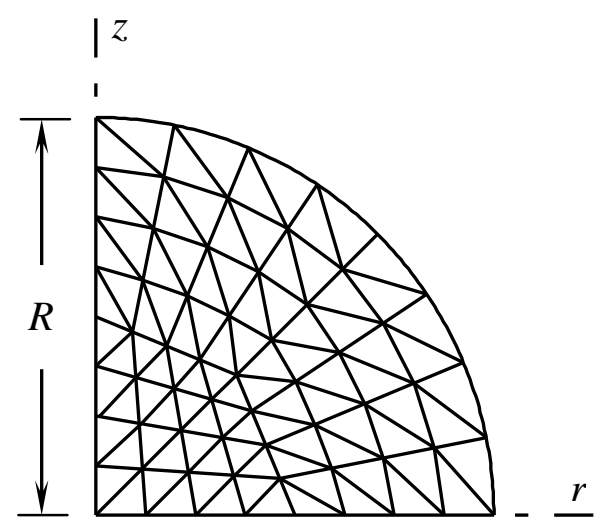

Figure 9. A semi-spherical domain with radius $R=1$ is meshed into 96 elements ( 8 elements along each coordinate axis). Boundary conditions derived from a spherical-wave solution are prescribed. 


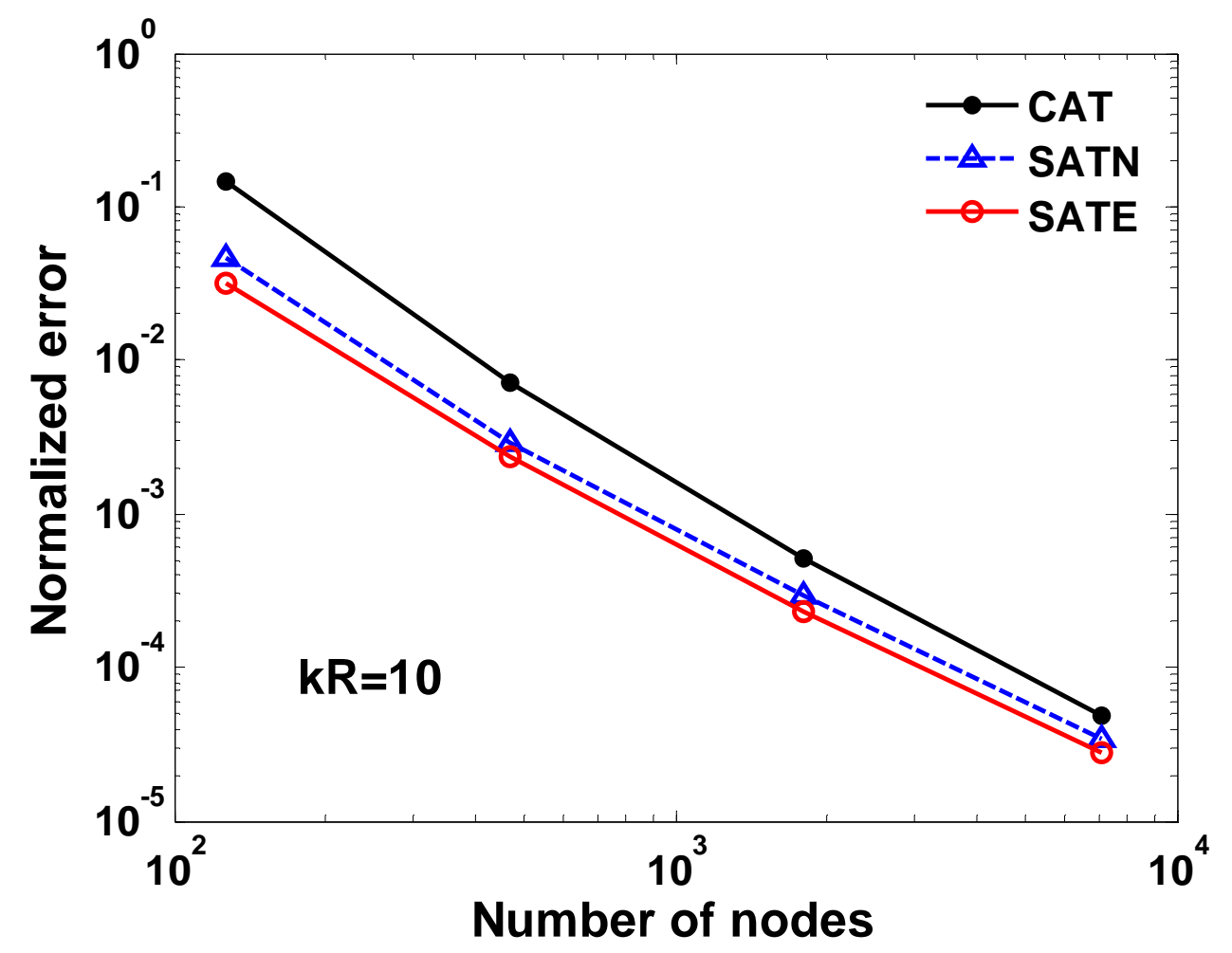

10(a)

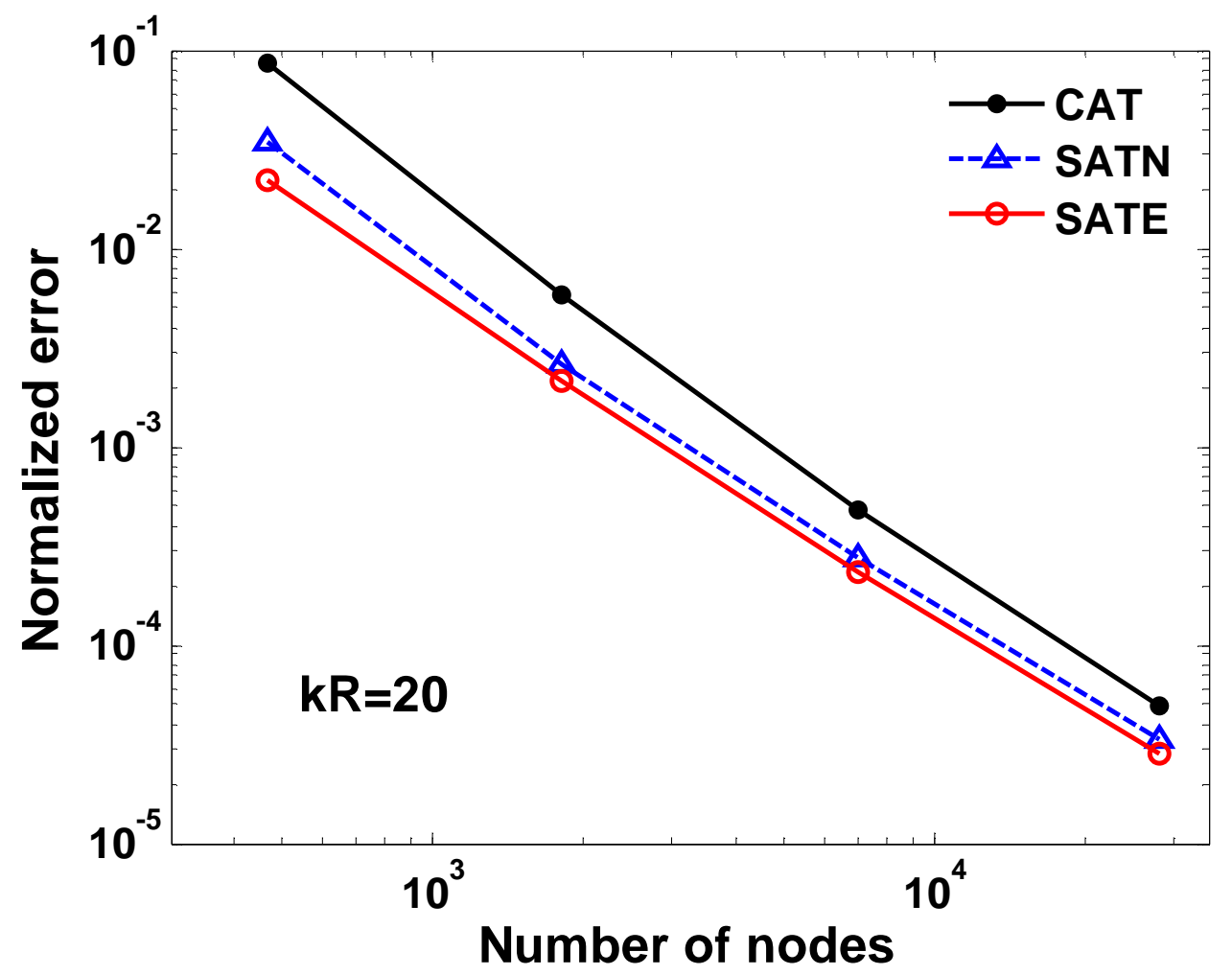

10(b)

Figure 10. Errors of the six-node elements in the spherical-wave problem. (a) $k R=10$ and (b) $k R=$ 20 are considered. $N_{e}$ is varied such that the number of nodal spacings per wavelength $N_{n}$ along the coordinate axis varies from $2.4 \pi$ to $9.6 \pi$. 